\title{
Simulated Microstructural Evolution and Design of Porous Sintered Wicks
}

\author{
Karthik K. Bodla \\ Cooling Technologies Research Center, \\ an NSF IUCRC, \\ School of Mechanical Engineering and \\ Birck Nanotechnology Center, \\ Purdue University, \\ West Lafayette, IN 47907
}

\section{Suresh V. Garimella ${ }^{1}$ Cooling Technologies Research Center, an NSF IUCRC, \\ School of Mechanical Engineering and Birck Nanotechnology Center, Purdue University, \\ West Lafayette, IN 47907 e-mail: sureshg@purdue.edu}

\begin{abstract}
Porous structures formed by sintering of powders, which involves material-bonding under the application of heat, are commonly employed as capillary wicks in two-phase heat transport devices such as heat pipes. These sintered wicks are often fabricated in an ad hoc manner, and their microstructure is not optimized for fluid and thermal performance. Understanding the role of sintering kinetics - and the resulting microstructural evolution-on wick transport properties is important for fabrication of structures with optimal performance. A cellular automaton model is developed in this work for predicting microstructural evolution during sintering. The model, which determines mass transport during sintering based on curvature gradients in digital images, is first verified against benchmark cases, such as the evolution of a square shape into an areapreserving circle. The model is then employed to predict the sintering dynamics of a sideby-side, two-particle configuration conventionally used for the study of sintering. Results from previously published studies on sintering of cylindrical wires are used for validation. Randomly packed multiparticle configurations are then considered in two and three dimensions. Sintering kinetics are described by the relative change in overall surface area of the compact compared to the initial random packing. The effect of sintering parameters, particle size, and porosity on fundamental transport properties, viz., effective thermal conductivity and permeability, is analyzed. The effective thermal conductivity increases monotonically as either the sintering time or temperature is increased. Permeability is observed to increase with particle size and porosity. As sintering progresses, the slight increase observed in the permeability of the microstructure is attributed to a reduction in the surface area. [DOI: 10.1115/1.4026969]
\end{abstract}

Keywords: sintering model, effective thermal conductivity, permeability, wicks, heat pipes, random packed beds, cellular automaton

\section{Introduction}

The unprecedented miniaturization of electronics and the advent of multicore architectures have led to continual increases in the thermal design power of microelectronics as predicted by the thermal Moore's law [1]. Many present-day, high-performance applications require cooling solutions that can handle local heat fluxes as high as $500 \mathrm{~W} \mathrm{~cm}^{-2}$ [2]. One solution for managing such high heat fluxes is to exploit phase change in pumped liquidcooling systems, as employed in two-phase jet impingement [3] and microchannel [4] heat sinks.

Heat pipes, vapor chambers, and two-phase capillary pumped loop devices capable of transporting heat over long distances without a substantial drop in temperature offer passive and compact cooling alternatives to pumped cooling systems [5,6]. These devices exploit capillary action generated by an internal porous material lining that enables passive transport of the working fluid. Owing to phase change and convective transport of the internal working fluid, the effective thermal conductivities of these devices are up to two or three orders of magnitude higher than for solid conductive materials of the same dimensions; these devices therefore find widespread application in thermal management [7]. Specifically, such two-phase heat transport devices are used widely for hot-spot cooling and heat spreading in the electronics cooling industry.

The two primary internal constituents of a heat pipe are the wick and the working fluid. Water, owing to its desirable thermophysical

\footnotetext{
${ }^{1}$ Corresponding author.

Contributed by the Heat Transfer Division of ASME for publication in the Journal of Heat Transfer. Manuscript received August 12, 2013; final manuscript received February 25, 2014; published online March 26, 2014. Assoc. Editor: Bruce L. Drolen.
}

properties and compatibility with the copper shell, remains the most commonly used working fluid in the temperature range typical of electronics cooling applications. Wicks, on the other hand, can be made from a variety of materials resulting in different structures. Wick types are classified as homogeneous wicks, such as those made of wrapped screen meshes and sintered metal powders, and as composite wicks; examples of the latter include composite screen wicks and screen-covered groove wicks. Due to the wide distribution of pore sizes, composite wicks provide high capillary pressure and high permeability, but are more difficult to manufacture [5]. The high production cost of composite wicks limits them to niche applications. Homogeneous wicks, on the other hand, are inexpensive and are widely employed.

Estimation of the transport properties of wick structures is essential for designing high-performance heat pipes. Dullien [8] provided an excellent review of experimental measurement techniques. In our previous work [9], a thorough characterization of the single-phase thermal performance of monoporous sintered copper powder wicks was performed via a microtomographybased direct numerical simulation. The limitations of present-day experimental methods and commonly used analytical expressions in characterizing single-phase heat transfer properties, such as effective thermal conductivity and permeability, was demonstrated. In follow-on work [10], several two-phase characteristics of interest, such as capillary pressure, effective pore radius, and evaporative heat fluxes, were estimated employing the volume of fluid method coupled with microtomography data. A detailed description of recent advances in analysis of thermal transport through porous sintered beds may be found in Ref. [11].

The various single-phase and two-phase heat transfer properties of a wick are all directly related to its microstructure, and desirable properties often compete with one another. For example, a 
smaller wick pore size promotes the desired higher capillary pressure; however, the smaller the pore size, the harder it is for fluid to flow through the wick, i.e., the permeability of the wick is reduced. Under these design constraints, optimizing the wick microstructure is a challenging problem, and calls for a fundamental model that can predict these various characteristics directly as functions of wick microstructure and fabrication processing parameters.

In the present work, we focus on characterizing the dependence of sintered powder wick transport properties on sintering kinetics. Sintering kinetics and modeling approaches are briefly reviewed. A cellular automaton model is first developed for predicting the microstructural evolution during sintering. Several two- and threedimensional configurations are considered, and the effective thermal conductivity and permeability of the wick structure are estimated. The model, which determines surface mass transport via curvature gradients, is first verified against benchmark, twodimensional cases. The performance of the numerical model is validated by comparing the neck size growth for a two-cylinder configuration against theory and experimental data available in the literature for sintering of cylindrical wires. Further, a twodimensional case corresponding to random packing of monosized cylinders is also considered from the literature, for prediction and comparison of simulated microstructural evolution and effective thermal conductivity. The numerical model is then employed to predict transport characteristics for various three-dimensional configurations of randomly dispersed spherical particles. Cases with varying porosity at a fixed particle size, and varying particle sizes at a fixed porosity, are considered to allow a delineation of the importance of porosity and particle size independently. Further, a realistic structure with particles sizes of a near-Gaussian distribution is also considered to depict a random, commercial wick.

\section{Numerical Modeling}

2.1 Sintering. A loose powder compact is sintered to form monolithic parts consisting of a collection of particles, interparticle connections (necks) and pore spaces, by heating the material to an elevated temperature below the melting point [12]. Interparticle bonding leads to a significant improvement in a number of structural and thermal characteristics of the part being sintered, such as strength, thermal conductivity, and capillary pressure [5]. Sintering may proceed via a number of mechanisms, such as surface diffusion, volume (lattice) diffusion, grain boundary diffusion, and evaporation-condensation diffusion mechanisms. German [12] provided a detailed description of these mechanisms; depending on characteristics such as particle size, temperature, and material properties, one or several of these could be the dominant diffusion mechanism.

Sintering is often described as occurring in three stagesinitial, intermediate, and final. The initial stage begins with a loose powder compact with particles in point contact with one another. Interparticle connections begin to form as material diffuses to the neck region from the rest of the surface, and the pores begin to round and start becoming circular. In the intermediate stage, pore-rounding continues, and the distinction between individual particles begins to fade. In this stage, the pores are still largely connected with each other [12]. In the final stage, the pore spaces become disconnected, and start to collapse if there are no interstitial trapped gases. Significant shrinkage and density change occurs and the individual grains (particles) are no longer distinguishable. Different diffusion mechanisms are dominant at these different stages; however, it must be noted that sintering is a continuous process and multiple diffusion mechanisms are active at any instant of time. Discrete stage distinctions have been made in the literature for the purpose of describing the sintering physics and modeling the microstructure evolution [12]. Figure 1 shows a typical sintered copper powder wick. The main distinguishing

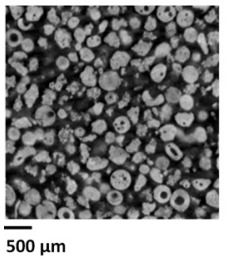

a)

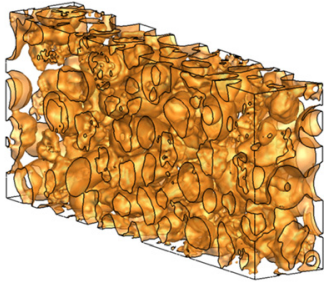

b)
Fig. 1 Microstructure of a sintered wick: (a) a 2D cross section obtained via X-ray microtomography, and $(b)$ corresponding 3D reconstructed microstructure, shown for a $250-355 \mu \mathrm{m}$ particle size wick from Ref. [9]

features of these realistic wicks that are not captured by a simple spherical packed bed are the interparticle neck shapes and the varied particle size distribution.

Sintering theories describing the idealized case of two uniformly sized spheres have been well developed [12-14]. Depending on the type of diffusion mechanism modeled, different rates of neck growth are obtained. In general, for the various diffusion mechanisms, the growth of neck size, $x$, relative to the particle size, $D$, temperature, $T$, and time of sintering, $t$, is described by the following functional form:

$$
x^{n}=\frac{C(T)}{D^{m-n}} t
$$

The exponents $m$ and $n$, and the factor $C(T)$, are assigned values depending on the specific diffusion mechanism [12]. Such theories have been widely used for describing material transport due to sintering for simple particle configurations in two and three dimensions. Attempts have also been made to include differentsized spheres to capture the effect of a particle size distribution (e.g., Ref. [15]). Some of the popular approaches for modeling sintering include the discrete element method (DEM), phase-field simulation, and Monte Carlo methods [15]. Martin et al. [16] employed DEM to simulate the sintering of metallic powders; a simple coarsening model was also employed to simulate densification. Monte Carlo methods have the advantages of being easy to code and easily extensible from two to three dimensions. Various microstructural evolution processes such as anisotropic grain growth, recrystallization, and Ostwald ripening have been successfully simulated employing Monte Carlo methods, as noted in Ref. [17]. In a series of works by Tikare et al. [18,19], solid-state sintering in two and three dimensions was successfully simulated. Later, in Ref. [17], a one-to-one comparison between the obtained results and the observed sintering of copper particles of sizes $40-60 \mu \mathrm{m}$ was presented. The model was observed to accurately predict the underlying physical processes, viz., densification and grain-growth [17].

Commercial sintered powder wick structures are typically composed of a collection of distributed particle sizes. It is infeasible to include large variations in particle sizes for predicting microstructural evolution with the aforementioned methods, owing to the high computational cost of treating material transport in a macroscopic volume down to the scale of the individual grains. Mathematically tracking a surface under the influence of its own dynamics is notoriously difficult. For the case at hand, the surface normal velocity is proportional to the divergence of the gradient of the curvature (in the coordinates of the surface); a nonlinear, fourth-order partial differential equation with moving boundary conditions results. As noted in Ref. [20], such calculations have either employed approximations or are solved using numerical techniques, and are always limited to simple cases. To address this challenge, Pimienta et al. [20] developed a model for simulating sintering evolution due to surface mass transport, driven by curvature differences; this approach is also employed in the 
current work. By estimating curvature of the surface indirectly from digital images, and relocating pixels (mass) based on the measured curvature differences, realistic cases of random particle collections in two and three dimensions were successfully simulated in Ref. [21]. In this approach, an atomically stepped surface is replaced with a continuous depiction, represented digitally by pixels. The size of a pixel is a small fraction of a particle, but far larger than atomic length scales. Several advantages of using digital images may be identified. Random particle shapes and sizes may be easily included. Physical quantities and transport characteristics may be readily computed based on images, either by employing the naturally discretized pixels as computational elements, or by employing image-based meshing techniques described in Refs. [9] and [22]. Curvature gradient-based sintering simulations have also been performed by Wakai and Aldinger [14] for simple cases such as sintering of two equally sized spheres. The energy minimization tool, surface evolver [23], was employed and the change in the interparticle neck was tracked for a variety of dihedral angles between the contacting spheres.

2.2 Cellular Automaton-Based Sintering Model. In this work, we employ the cellular automaton-based sintering model originally proposed in [20], and later extended to three dimensions in Ref. [21]. The model is only described briefly here, and readers are referred to Refs. [20] and [21] for details. The core of the algorithm is appropriate curvature estimation. Curvature of a surface may be readily estimated when the shape of the surface is known analytically; however, for the case of random particle configurations, such a description is not always possible. Furthermore, when irregularly shaped particles are considered, curvature estimation becomes even more challenging. To overcome these limitations, a pixel-counting method was suggested for indirectly calculating the curvature, based on a method originally proposed by Vicsek [24]. In this method, an imaginary box is drawn centered around the pixel for which curvature is being estimated. Curvature is calculated as being proportional to the number of void (nonmaterial) pixels falling inside the box [20]. By considering simple cases of circles and spheres, it was shown that such a pixel count follows a linear relationship with respect to the actual value of curvature, with the slope of the line varying with the size of the pixel counting box [20]. Hence, as long as only the relative curvatures are important and not the exact values, as in the case of curvature-driven mass transport, such estimates are useful.

The domain consists of only two phases, the material phase and the void (air) phase. In each iteration of the algorithm, curvature is estimated for interface pixels on the material side, and the pixels are ordered in decreasing order of these curvature values. From these, a prescribed number, $n$, of the highest curvature pixels are selected and removed (reassigned the void phase). If there are multiple pixels with the same curvature cutoff value, selection for movement is randomized. In the next step, the curvature value is estimated for the void-side interface pixels. These pixels are then arranged in increasing order of curvature values, and now, $n$ lowest curvature-valued pixels are selected and assigned the material phase [20]. At the end of an iteration, $n$ highest-curvature solid pixels have thus been selected and moved to $n$ lowestcurvature void spaces. The procedure is repeated for the desired number of iterations, or until an equilibrium shape is obtained. This algorithm removes mass in a conserved fashion, i.e., the total number of solid pixels remains constant throughout in the direction of the curvature gradient. Furthermore, the algorithm is easy to implement, readily extensible to three dimensions, and can model random configurations easily.

In this work, we employ this algorithm for predicting microstructural evolution during sintering. Subsequently, the microstructures are employed to estimate transport characteristics, effective thermal conductivity, and permeability. Since digital images are naturally discretized, image-based finite difference methods (FDM) may be employed for transport property prediction. Alternatively, the images may be transformed into a computational mesh to allow analysis with commercial solvers. In this work, we adopt 2D image-based finite difference codes developed by Bohn and Garboczi [25] for calculating effective thermal conductivity of 2D microstructures. For 3D microstructures, we employ a similar approach based on a 3D finite difference code for computing effective thermal conductivity, while image-based mesh generation and subsequent computational fluid dynamics analysis, as described in detail in Refs. [9] and [22], is employed for estimating permeability. Figure 2 shows a sample workflow for estimating transport properties as a function of the extent of sintering, beginning with a user-defined particle size distribution for the initial random particle bed.

\section{Results and Discussion}

Qualitative microstructural evolution results are first discussed for two 2D configurations, a square shape transforming to an areapreserving circle, and neck formation and evolution for the case of two circles initially in point contact. A two-dimensional case consisting of randomly distributed monosized cylinders that are initially in point contact is also considered for which qualitative as well as quantitative comparison of the present computations is made against similarly computed data from the literature. Results

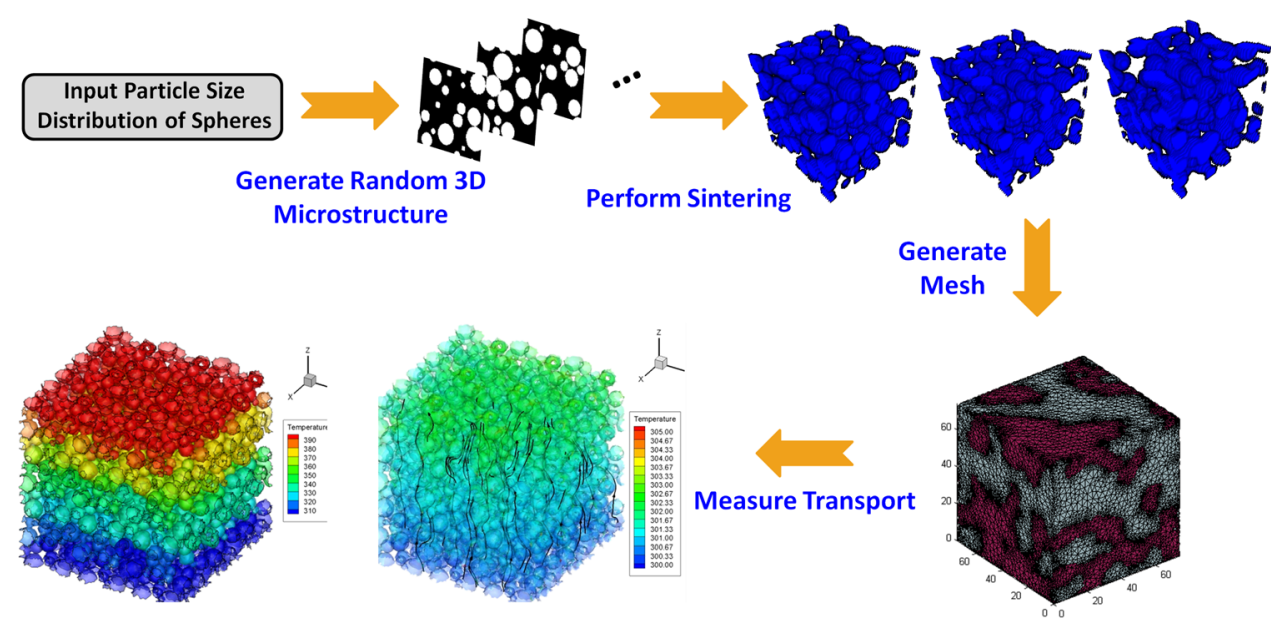

Fig. 2 The workflow employed in the current work for estimating transport characteristics for a wick with user-defined particle size distribution is shown 
for effective thermal conductivity that quantify the influence of sintering neck size are presented.

Qualitative and quantitative evolution of random, 3D microstructures is then presented. Cases corresponding to fixed particle size (varying porosity), and fixed porosity (varying particle size) are considered to allow a delineation of porosity and particle size independently. The influence of these parameters on transport properties of interest, viz., effective thermal conductivity and permeability, is quantified via direct computations based on the generated 3D microstructures. Furthermore, a realistic case consisting of a nearly Gaussian distribution of particle sizes is investigated for demonstrating the flexibility of the framework developed here. For the various cases, the influence of sintering neck size on the effective thermal conductivity and permeability are quantified.

\subsection{D Microstructural Evolution}

3.1.1 Area-Preserving Square to Circle Evolution. The first 2D case considered corresponds to evolution of a square to an area-preserved circle. A square has large curvature gradients; the curvature at the sharp corners is significantly higher compared to that along the flat sides. Owing to these curvature differences, mass (pixels) migrates from the corners to the flatter portions. At equilibrium, we obtain a circle for which there are zero curvature gradients. It may be noted that as we employ the pixel-counting box algorithm for estimating curvature, curvature differences beyond the size of the box cannot be accounted for, as also noted in Ref. [20]. This calls for a large counting box. On the other hand, when the size of the pixel-counting box is large, nonlocal effects may be undesirably included; i.e., when the size of the pixel-counting box is of the order of the size of the particles themselves, pixels from the surfaces of the neighboring particles are counted. It was noted in Ref. [20] that a counting box of size 3 or 5 pixels is sufficient for all practical purposes. We employ a counting box of size 5 pixels in this work for particle diameters resolved by $\sim 10-25$ pixels. Figure 3 shows the qualitative microstructural evolution of a square to a circle. This case corresponds to a square of $30 \times 30$ pixels, with $n=1$ pixels removed per iteration. It may be noted that the corner pixels with the highest curvature are the first to be relocated. For this case, the equilibrium circle shape was obtained in approximately 100 iterations.

3.1.2 Sintering of Two Cylinders in Point Contact. A widely studied case for model validation is the sintering of two cylinders in point contact. Beginning with the contacting cylinders, owing to curvature differences between the outer surface of the particles and the neck (interparticle contact) regions, mass (pixel) migration occurs. According to Eq. (1), as the temperature increases, the time to reach a particular neck size $x$ decreases [12]. In our
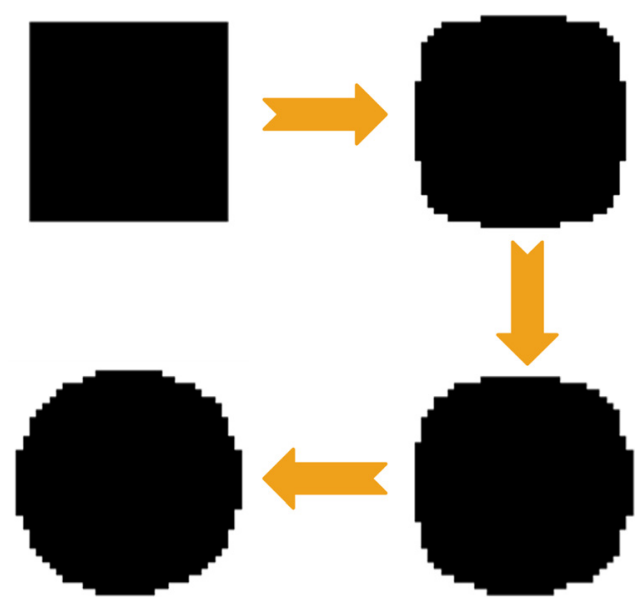

Fig. 3 Microstructure evolution of a square to an areapreserved circle, shown for a square with $30 \times 30$ pixels algorithm, the number of pixels migrating per iteration, $n$, is directly proportional to temperature, and the number of iterations is proportional to time. To verify this, the two-cylinder scenario is considered and the number of iterations necessary for the neck size to reach a specified value of $x / D=0.3$ is calculated for different values of $n$. As shown in Fig. 4(a), the number of iterations required decreases as $n$ increases. Moreover, the iteration count (time) versus $1 / n$ relationship is linear, in accordance with Eq. (1). This verifies that the effect of temperature is correctly captured via the number of pixels migrating per iteration. Further, Fig. 4(b) shows evolution of nondimensional neck size as a function of iteration number for multiple $n$ values. It may be observed that the slope of the curves is independent of $n$, confirming that the morphology of neck growth versus time is unaffected by $n$, as also observed in Ref. [26]. At higher values of $n$ (temperature), the time to reach a particular neck size decreases; however, the neck size-to-time relationship remains the same, as also observed in the experiments of Ref. [26].

As a second verification step, the rate of neck growth for the two-particle configuration is compared against the experiments of Alexander and Balluffi [26]. In Ref. [26], sintering of cylindrical copper wires of $128 \mu \mathrm{m}$ diameter was studied experimentally. Sintering stages of neck formation and growth, pore smoothening and rounding, grain growth, pore isolation, and closure were observed; neck evolution was measured for sintering at various temperatures in the range $900-1075^{\circ} \mathrm{C}$. It was observed that neck size raised to the fifth power increased linearly with time [26]. In our simulations, this same linear relationship with number of iterations (time) was observed for $(x / D)^{4.5}$, as shown in Fig. 4(c). This further validates that the rate of neck growth is being modeled correctly.

Having validated the sintering model, the effective thermal conductivity of the two-cylinder configuration is estimated. For this, a temperature gradient is imposed across the ends of the sample and the amount of heat conducted is measured to calculate $k_{\text {eff }}$ as [9]

$$
k_{\mathrm{eff}}=\frac{-\int \mathbf{J} . d \mathbf{A}}{\left(\frac{\partial T}{\partial x_{i}}\right) A}
$$

The calculations are performed on a grid discretized directly at the pixel centroids in the 2D image. The open-source FDM codes of Bohn and Garboczi [25], developed for calculating effective electrical conductivity, were employed in this work by exploiting the electrical-thermal conduction analogy. Figure 5 shows representative results obtained for a copper-water material combination. It is observed that the neck region is a constricting, highresistance region, and the effective thermal conductivity improves as the neck develops. Most of the recommended correlations relate effective thermal conductivity of sintered powder materials to porosity as the lone independent parameter. As noted in our previous work [9], and from Fig. 5(b), effective thermal conductivity varies for a fixed value of porosity; new correlations capturing the effect of neck size and not just porosity, are desirable.

3.1.3 Sintering of a Random Collection of Circular Particles. As a final 2D case, a randomly spaced collection of equal-sized disks of 15 pixels radius is considered. The initial microstructure is adopted from Ref. [15], and the microstructural evolution is shown qualitatively in Fig. 6(a). It may be observed in the first several iterations that the pore space starts to round out and sintering necks begin to form. As iterations progress further, the pores round further and then eventually disappear. These observations are consistent with actual experimental observations typically made in sintering experiments, as discussed in Refs. [12] and [15]. It may be noted that a few of the particles along the edges were sharply cut off in the initial image; as nonperiodic boundary conditions were employed in this study, these particles had the 


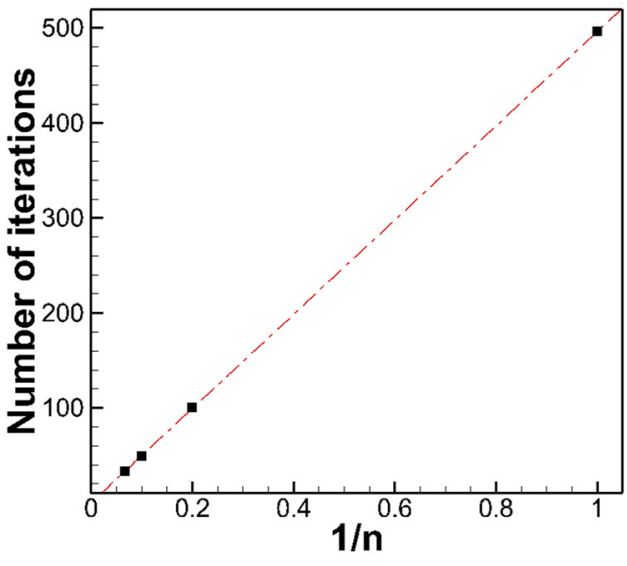

a)

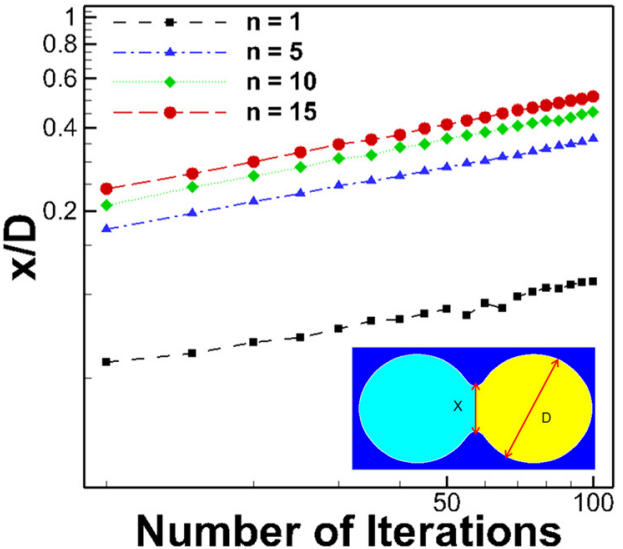

b)

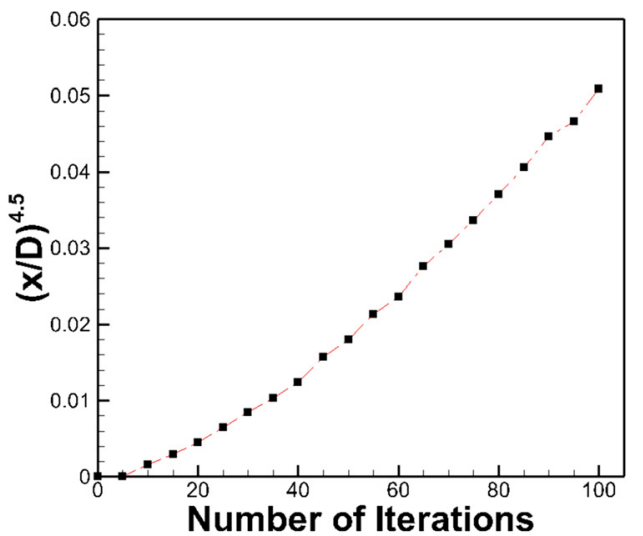

c)

Fig. 4 Sintering kinetics for a two-cylinder sintering scenario: (a) number of iterations required to reach a constant neck size, and $(b)$ relative neck size growth as a function of sintering iterations for various values of $n$. Also shown in $(c)$ is the typical power law behavior of neck size growth, as observed in experiments and predicted by theory (Eq. (1)).

highest initial curvature and were hence among the first to sinter (smoothen). Also shown for comparison in Fig. 6(a) are snapshots from Ref. [15], in which a phase field approach was employed to model sintering.

Employing the same procedure as described for the twocylinders case, effective thermal conductivity was estimated in both the horizontal and vertical image directions for this case. In Fig. 6(b), representative values for the original microstructure (zero relative change in surface area) along with three intermediate stages are plotted as a function of change in overall surface area relative to the area of the starting microstructure. Also shown in Fig. 6(b) is similarly computed results for the microstructures

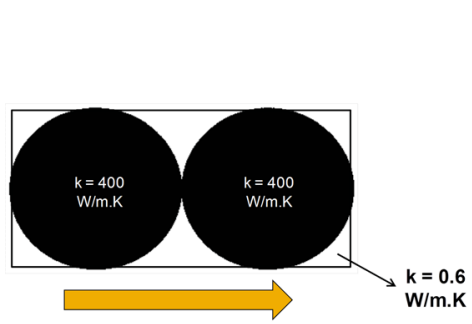

a)

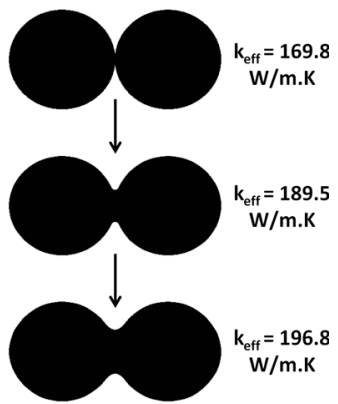

b)
Fig. 5 Effective thermal conductivity calculation for the two-cylinder sintering scenario: (a) problem setup, and (b) computed values for initial and intermediate stages from Ref. [15] shown in Fig. 6(a). Very good agreement is observed between the present data and those from Ref. [15], which further validates the sintering model developed. Again, it may be observed that thermal conductivity improves drastically as the sintering neck develops-as little as a $20 \%$ change in surface area leads to $\approx 4-7$ times improvement in effective thermal conductivity, relative to that of the starting microstructure consisting of cylinders in point contact. Shown in Fig. 6(c) is the decrease in overall surface area of the compact relative to its initial surface area (surface area of pre-sintered compact) as a function of sintering iterations, for three different values of pixels migrating per iteration, $n$. As expected, at any value of $n$, the surface area decreases as iterations progress, as sintering is a surface areaminimizing phenomenon. Also, as the value of $n$ increases, the surface area decreases rapidly, and vice versa, i.e., necks form and grow more rapidly at higher temperatures, and surface area thereby decreases at a relative higher rate.

3.2 3D Microstructural Evolution. Commercial sintered wicks consist of a random collection of particles. In this study, apart from the ideal and random 2D microstructures discussed earlier, several 3D, random microstructures consisting of spherical particles are also considered. The evolution of the microstructure during sintering is predicted. In the following, qualitative results of microstructural evolution are presented, along with predictions of effective thermal conductivity and permeability.

3.2.1 3D Microstructure Generation. For the initial random packed bed configuration, we use the random particle generator described in Ref. [27]. Beginning with the largest-sized particles, 


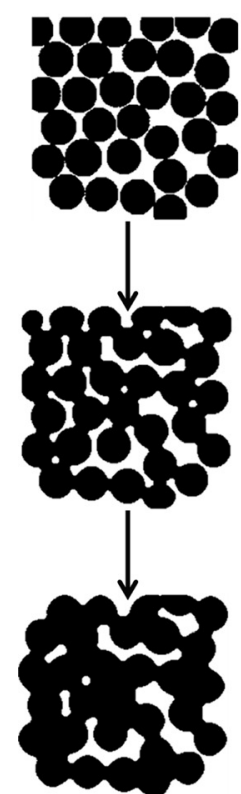

Present Study
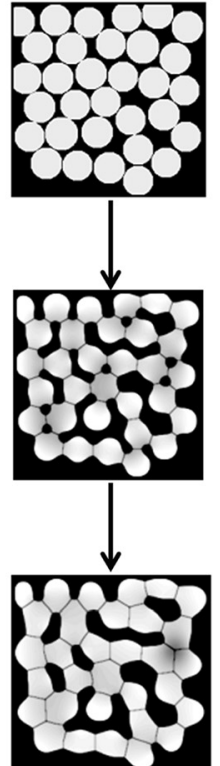

Phase field simulation (Kumar,2011)

a)

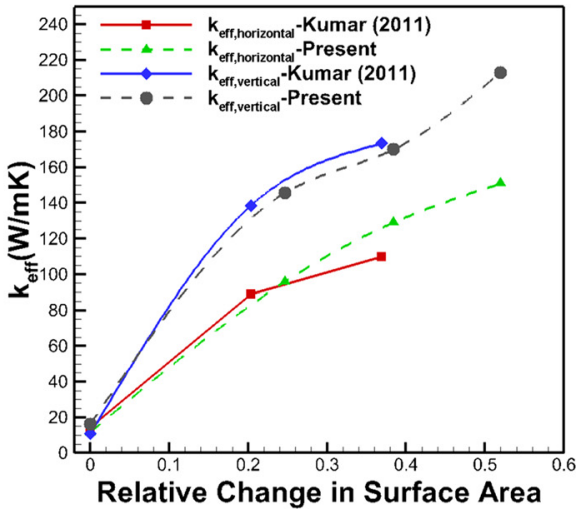

b)

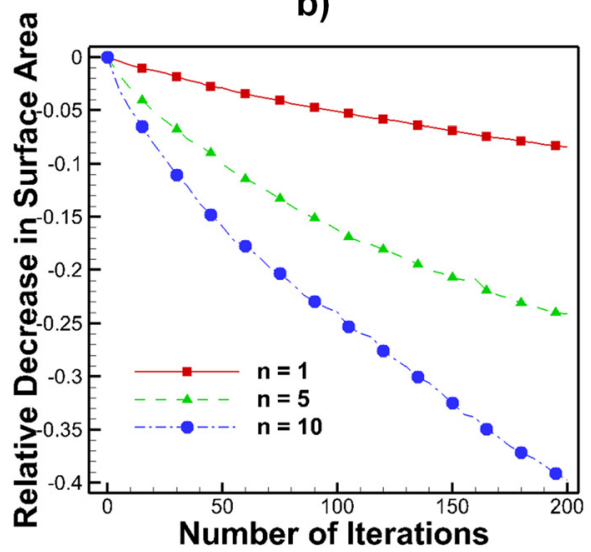

c)

Fig. 6 (a) Microstructure evolution and comparison with phase-field simulations of [15], (b) predictions of effective thermal conductivity, and (c) sintering kinetics for a random 2D circular particle bed

a user-specified distribution of particle sizes (number and radius of each particle) is placed iteratively. Porosity may be controlled indirectly by varying the particle radius and count for each particle type; however, the random generator lacks a shaking algorithm, and porosities lower than approximately $56 \%$ (i.e., particle fractions greater than $44 \%$ ) were difficult to obtain. Tightly packed structures are often generated using shaking algorithms to lower the energy of the system, and thus pack the particles closely.

Further, this algorithm generates the microstructure with periodic boundary conditions, i.e., when a particle coincides with the boundary, the part of the particle falling out of the domain is placed at the opposite side of the boundary [27]. Following random particle placement, the generator also has an optional flocculation step, which may be used to control the number of clusters (connected entities) in the microstructure. In the present study, we generate and flocculate the structure such that the resulting structure is a single connected entity. Periodic boundary conditions are again employed during flocculation [27], and hence, the generated 3D microstructure typically still contains many loose particles. An image-based mesh generation process is employed to generate computational meshes from the 3D image data (discussed, for example, in Ref. [9]), but generation of periodic meshes is not always possible. Employing such meshes made from microstructures consisting of loose particles is thus not viable for computing effective thermal conductivity. To circumvent this issue, we employ the 3D finite difference code of Bohn and Garboczi [25], which directly operates on pixel centers as nodes and uses periodic boundary conditions by layering a shell of imaginary finite difference nodes around the real ones. The temperature values at those imaginary nodes are obtained from the corresponding real ones by invoking the periodicity condition, and the discretized set of equations corresponding to the Laplacian equation for energy transport are solved via a conjugate gradient method [25]. For a detailed description of the approach and validation cases, readers are referred to Bohn and Garboczi [25]. We continue to use the image-based mesh generation technique to compute permeability, however, and employ the commercial finite volume solver, Fluent [28]. We retain just the pore space of the microstructures for this computation and generate the corresponding volumetric meshes. Employing this approach, several 3D microstructures are generated, and their properties computed.

3.2.2 Effect of Particle Size. In our previous study, effect of particle size on the transport characteristics of the wicks was analyzed by considering commercial wicks manufactured at $\sim 63 \%$ porosity [9]. It was observed that the effective thermal conductivity increased as the particle size decreased. This was attributed to increased interparticle necking at relatively smaller particle sizes. Similarly, the smallest particle size samples demonstrated the lowest permeability and vice versa, which was attributed to the corresponding decrease in pore size with decreasing particle size. In this study, we consider three particle sizes, viz., $110 \mu \mathrm{m}$, $170 \mu \mathrm{m}$, and $250 \mu \mathrm{m}$ generated at a porosity of $\sim 60-62 \%$. Figure 7 shows representative microstructures at various stages of sintering, along with the computed values of effective thermal conductivity plotted as a function of change in surface area relative to the original surface area. The effective thermal conductivity values were computed numerically by imposing a temperature gradient and determining the average heat flux, as described in Ref. [25] for the case of effective electrical conductivity, and the values reported in this work are averaged over the three coordinate 

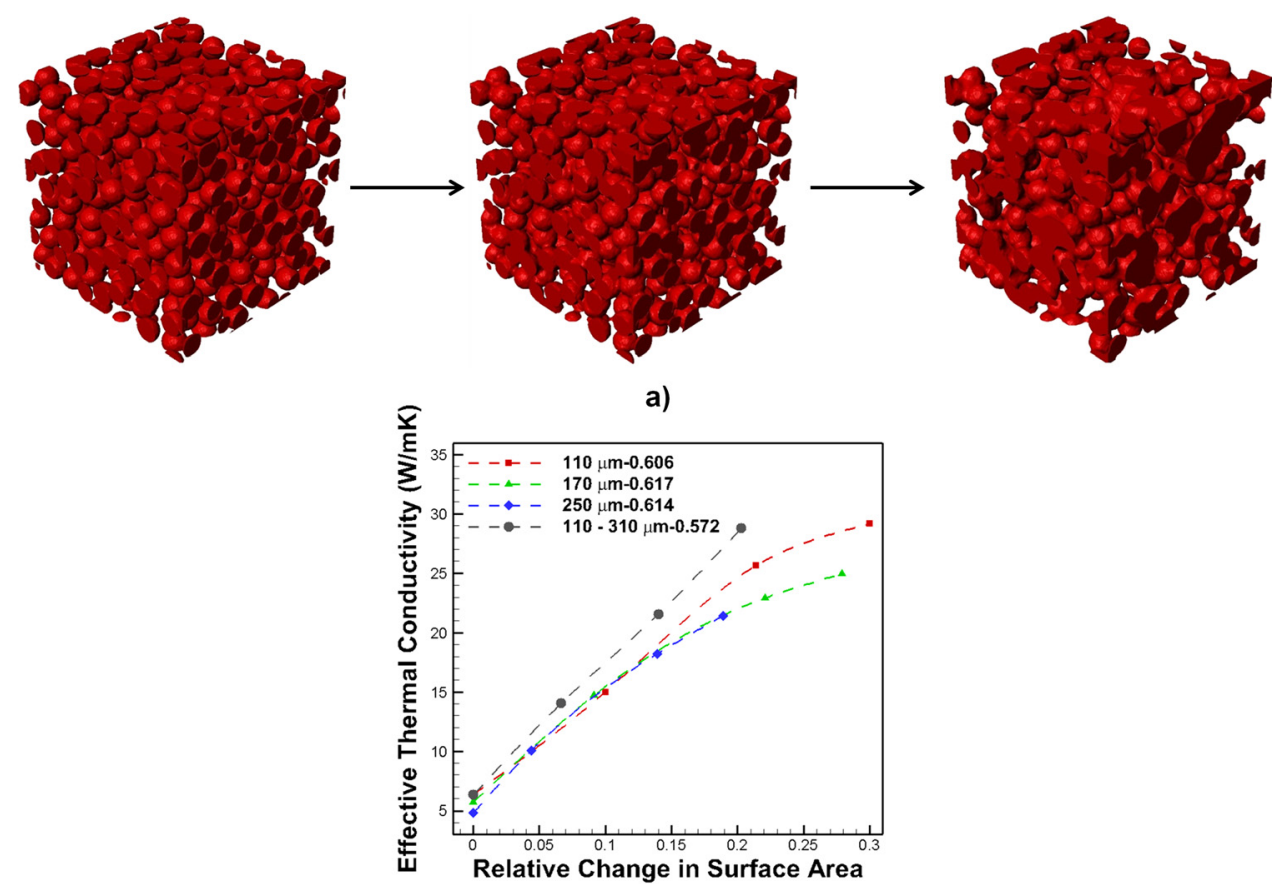

b)

Fig. 7 Effect of particle size on sintering of random, 3D spherical powder compacts: (a) microstructure evolution for sample made up of $170 \mu \mathrm{m}$ particles, and (b) effective thermal conductivity as a function of change in surface area relative to the initial microstructure

directions. Convergence of the numerical procedure is tracked by monitoring the evolution of effective thermal conductivity as a function of iterations-the solution is deemed converged if the effective thermal conductivity values along all the directions remained constant with iterations. As indicated in Fig. 8, such a converged solution was obtained within about 100 iterations for all the cases investigated in this study. For all particle sizes, effective thermal conductivity increases as surface area decreases, in an approximately linear fashion. Further, effective thermal conductivity is observed to increase as porosity decreases, as expected. Also, shown in Fig. 7 is a test case in which a particle size range $(110-310 \mu \mathrm{m})$ with nearly Gaussian distribution were

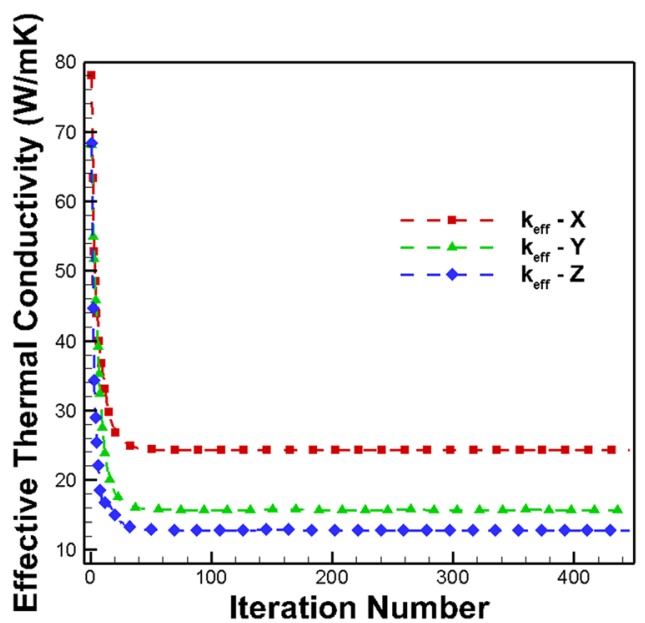

Fig. 8 Convergence history of the 3D finite difference code from Ref. [25] employed in this work for calculating effective thermal conductivity of 3D microstructures directly. The solution may be assumed converged after approximately 100 iterations for all cases considered in this study. considered. This case had a porosity of $\sim 57 \%$, and demonstrated the highest effective thermal conductivity.

Permeability and Ergun's coefficient values were also computed. For these computations, velocity inlet and pressure outlet boundary conditions are prescribed on opposite ends of the computational domain, while the lateral sides have symmetry conditions imposed. The pore velocity is varied, and the observed pressure drop is correlated to velocity, employing Darcy's law

$$
\left(-\frac{\partial P}{\partial x}\right)=\frac{\mu}{K} U+\frac{\rho C_{E}}{\sqrt{K}} U^{2}
$$

Further details on computing permeability of random porous media and employing meshes generated via $3 \mathrm{D}$ images may be found in Refs. [22] and [9]. Table 1 shows permeability and Ergun's coefficient values for the various cases considered to analyze the influence of particle size. Similar to the observations for effective thermal conductivity, permeability is observed to increase, albeit only slightly, with the extent of sintering. Ergun's coefficient values, on the other hand, were observed to be more or less constant with sintering, and only average values are reported. Such an increase in permeability with sintering may be attributed to two factors. First, sintering is a surface area-reducing phenomenon; with relatively small amounts of sintering, the pore size remains essentially constant while the surface area decreases, which leads to an increase in permeability. Along similar lines, as the particles begin to sinter, they move closer to one another, and since we employ a fixed bounding box, such movement of the particles near the boundaries opens up the pore space, as may be observed from Fig. 7, further contributing to increased permeability. Similar observations were also made by Espinosa [29], who experimentally measured effective thermal conductivity, permeability, and capillary pressure for sintered copper powder samples as a function of sintering time. A variety of sintering temperatures and particle sizes were considered, and it was noted that effective thermal conductivity generally increased as sintering time increased. Permeability, on the other hand, was observed to remain relatively independent of sintering conditions, but 
Table 1 Permeability and Ergun's coefficient predictions, and comparison with Kozeny-Carman correlation, shown for the case of microstructures with constant porosity

\begin{tabular}{|c|c|c|c|c|c|}
\hline $\begin{array}{l}\text { Case } \\
\text { (Diameter-Porosity) }\end{array}$ & $\begin{array}{l}\text { Effective particle } \\
\text { diameter, } D_{P}, \\
\text { Eq. }(5)(\mu \mathrm{m})\end{array}$ & $\begin{array}{l}\text { Permeability, } \\
K\left(\mathrm{~m}^{2}\right)\end{array}$ & $\begin{array}{l}\text { Permeability } \\
\text { from K-C, } \\
\text { Eq. (4) }\left(\mathrm{m}^{2}\right)\end{array}$ & $\begin{array}{c}\text { Relative } \\
\text { percentage } \\
\text { difference (\%) }\end{array}$ & $\begin{array}{c}\text { Ergun's } \\
\text { coefficient } \\
C_{E}\end{array}$ \\
\hline $110 \mu \mathrm{m}-0.61$ & $\begin{array}{l}113 \\
125 \\
143 \\
160\end{array}$ & $\begin{array}{l}1.14 \times 10^{-10} \\
1.33 \times 10^{-10} \\
1.69 \times 10^{-10} \\
1.80 \times 10^{-10}\end{array}$ & $\begin{array}{l}1.21 \times 10^{-10} \\
1.50 \times 10^{-10} \\
1.97 \times 10^{-10} \\
2.49 \times 10^{-10}\end{array}$ & $\begin{array}{l}6.12 \\
12.4 \\
16.4 \\
38.3\end{array}$ & 0.22 \\
\hline $170 \mu \mathrm{m}-0.62$ & $\begin{array}{l}162 \\
178 \\
208 \\
224\end{array}$ & $\begin{array}{l}2.43 \times 10^{-10} \\
2.61 \times 10^{-10} \\
3.11 \times 10^{-10} \\
3.50 \times 10^{-10}\end{array}$ & $\begin{array}{l}2.80 \times 10^{-10} \\
3.38 \times 10^{-10} \\
4.62 \times 10^{-10} \\
5.40 \times 10^{-10}\end{array}$ & $\begin{array}{l}15.1 \\
29.6 \\
48.7 \\
54.4\end{array}$ & 0.248 \\
\hline $250 \mu \mathrm{m}-0.61$ & $\begin{array}{l}237 \\
248 \\
276 \\
293\end{array}$ & $\begin{array}{l}5.18 \times 10^{-10} \\
5.47 \times 10^{-10} \\
6.73 \times 10^{-10} \\
7.42 \times 10^{-10}\end{array}$ & $\begin{array}{l}5.82 \times 10^{-10} \\
6.37 \times 10^{-10} \\
7.85 \times 10^{-10} \\
8.85 \times 10^{-10}\end{array}$ & $\begin{array}{l}12.3 \\
16.5 \\
16.6 \\
19.2\end{array}$ & 0.184 \\
\hline $110-310 \mu \mathrm{m}-0.57$ & $\begin{array}{l}230 \\
246 \\
267 \\
288\end{array}$ & $\begin{array}{l}3.72 \times 10^{-10} \\
4.14 \times 10^{-10} \\
5.18 \times 10^{-10} \\
6.59 \times 10^{-10}\end{array}$ & $\begin{array}{l}3.60 \times 10^{-10} \\
4.14 \times 10^{-10} \\
4.88 \times 10^{-10} \\
5.68 \times 10^{-10}\end{array}$ & $\begin{array}{r}3.16 \\
0.04 \\
-5.81 \\
-13.7\end{array}$ & 0.37 \\
\hline
\end{tabular}

increased suddenly as the wick was sintered further and began to detach from the bounding walls. Further, permeability was observed to be strongly dependent on the particle/pore size at a fixed porosity; permeability increased as average particle size increased [29]. Also shown in Table 1 are the permeability values predicted by the popular Kozeny-Carman correlation defined below, along with percentage difference relative to the current computations

$$
\frac{D_{p}^{2} \varepsilon^{3}}{150(1-\varepsilon)^{2}}
$$

It may be noted that $D_{p}$ is an effective particle diameter and is computed as follows:

$$
D_{p}=\frac{6(1-\varepsilon) V_{T}}{A_{s f}}
$$

where $V_{T}$ is the total volume of the sample including the pore and the material phases, and $A_{s f}$ is the interstitial surface area [11]. As sintering progresses, the surface area decreases, and this leads to an increase in particle size $D_{p}$ as also indicated in Table 1 . Also, as may be noted from Table 1, the Karman-Cozeny correlation gives a relatively good estimate of permeability at small values of sintering, but as sintering progresses, the correlation becomes less and less accurate. Further, for any amount of sintering and any particle size, the correlation is observed to over-predict permeability in general. Only for the case of a mixture of particle sizes were the permeability values from the present computations slightly
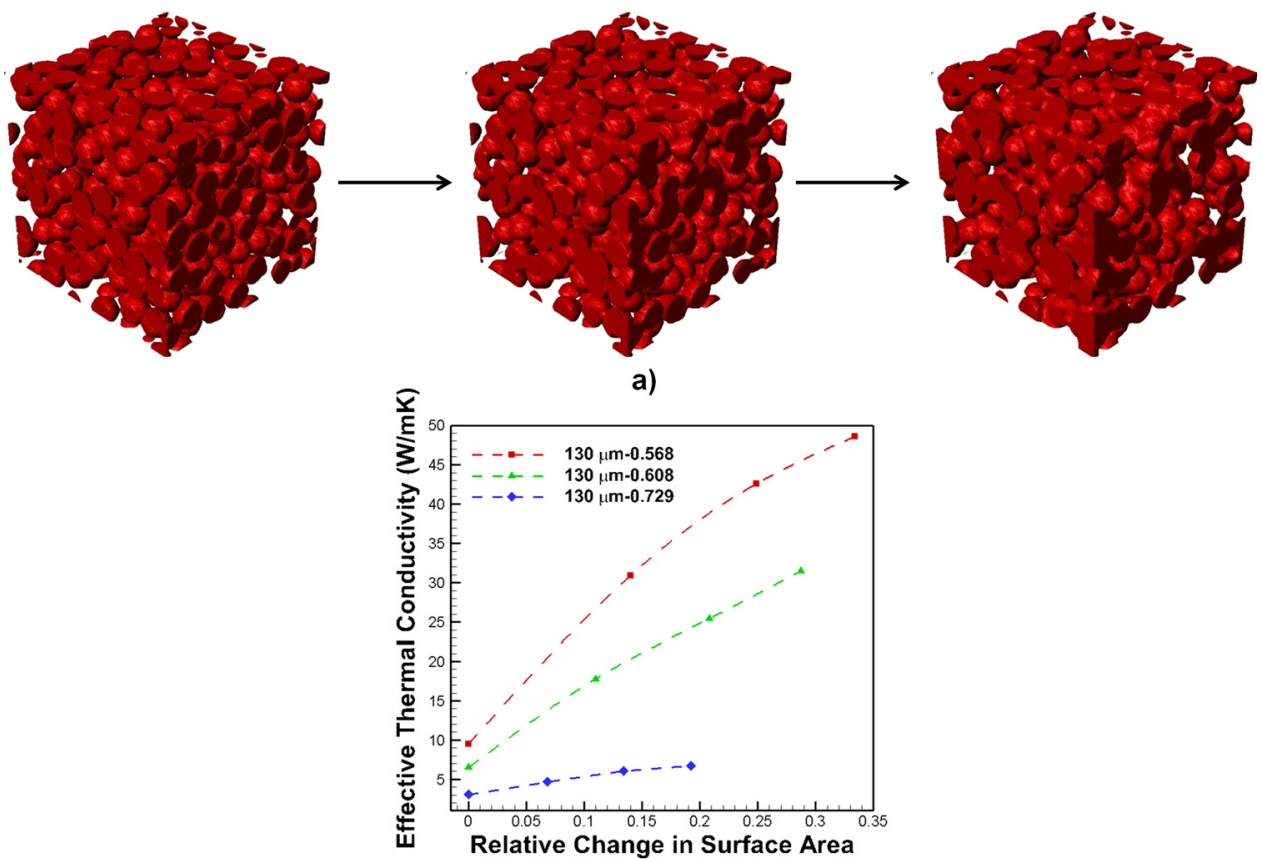

b)

Fig. 9 Effect of porosity on sintering of random, 3D spherical powder compacts: (a) microstructure evolution for the case of $57 \%$ porosity, and (b) effective thermal conductivity as a function of relative change in surface area 
Table 2 Permeability and Ergun's coefficient predictions, and comparison with Kozeny-Carman correlation, shown for the case of microstructures with constant particle size

\begin{tabular}{|c|c|c|c|c|c|}
\hline $\begin{array}{l}\text { Case } \\
\text { (Diameter-porosity) }\end{array}$ & $\begin{array}{c}\text { Effective particle } \\
\text { diameter, } D_{P}, \\
\text { Eq. }(5)(\mu \mathrm{m}),\end{array}$ & $\begin{array}{l}\text { Permeability, } \\
\qquad K\left(\mathrm{~m}^{2}\right)\end{array}$ & $\begin{array}{l}\text { Permeability } \\
\text { from K-C, } \\
\text { Eq. (4) }\left(\mathrm{m}^{2}\right)\end{array}$ & $\begin{array}{c}\text { Relative percentage } \\
\text { difference } \\
(\%)\end{array}$ & $\begin{array}{c}\text { Ergun's } \\
\text { coefficient, } \\
C_{E}\end{array}$ \\
\hline $130 \mu \mathrm{m}-0.57$ & $\begin{array}{l}125 \\
146 \\
167 \\
188\end{array}$ & $\begin{array}{l}9.73 \times 10^{-11} \\
1.21 \times 10^{-10} \\
1.56 \times 10^{-10} \\
1.84 \times 10^{-10}\end{array}$ & $\begin{array}{l}1.02 \times 10^{-10} \\
1.38 \times 10^{-10} \\
1.82 \times 10^{-10} \\
2.31 \times 10^{-10}\end{array}$ & $\begin{array}{l}4.83 \\
14.3 \\
16.4 \\
25.3\end{array}$ & 0.45 \\
\hline $130 \mu \mathrm{m}-0.61$ & $\begin{array}{l}126 \\
142 \\
159 \\
177\end{array}$ & $\begin{array}{l}1.41 \times 10^{-10} \\
1.56 \times 10^{-10} \\
1.94 \times 10^{-10} \\
2.50 \times 10^{-10}\end{array}$ & $\begin{array}{l}1.55 \times 10^{-10} \\
1.95 \times 10^{-10} \\
2.47 \times 10^{-10} \\
3.06 \times 10^{-10}\end{array}$ & $\begin{array}{l}10.1 \\
25.5 \\
27.3 \\
22.2\end{array}$ & 0.420 \\
\hline $130 \mu \mathrm{m}-0.73$ & $\begin{array}{l}125 \\
134 \\
144 \\
154\end{array}$ & $\begin{array}{l}4.65 \times 10^{-10} \\
4.85 \times 10^{-10} \\
5.21 \times 10^{-10} \\
6.05 \times 10^{-10}\end{array}$ & $\begin{array}{l}5.43 \times 10^{-10} \\
6.26 \times 10^{-10} \\
7.26 \times 10^{-10} \\
8.33 \times 10^{-10}\end{array}$ & $\begin{array}{l}16.7 \\
29.0 \\
39.2 \\
37.8\end{array}$ & 0.217 \\
\hline
\end{tabular}

higher relative to those computed via the correlation, as indicated in Table 1. Similar observations were also made in our previous work, in which we computed permeability values for real, random wick structures [9].

3.2.3 Effect of Porosity. The effect of porosity was analyzed by considering three microstructures with porosities of $57 \%, 61 \%$, and $73 \%$, all with randomly dispersed spherical particles of size $130 \mu \mathrm{m}$. Figure 9 shows a few representative 3D microstructure images, along with effective thermal conductivity data plotted as a function of change in surface area relative to the surface area of the original compact for the porosities investigated. As expected, effective thermal conductivity decreases as porosity increases. As sintering proceeds, the overall surface area of the compact decreases and the necks grow in size, owing to which, thermal conduction improves. As shown in Table 2, permeability is observed to be a direct function of porosity. An increase in porosity at a fixed particle size enlarges the pore space (pore size increases) and increases the permeability. Furthermore, similar to the previous case of fixed porosity, permeability for this case was also observed to increase slightly as a function of sintering time, which may again be attributed to the reduction in surface area and opening up of pore space near the boundaries, as evident from Fig. 9.

\section{Conclusions}

In this work, microstructure development during sintering is modeled via a cellular automaton algorithm. This algorithm is validated against theory and past experimental data for sintering of cylindrical wires. Several 2D cases are considered for tracking microstructure evolution, and understanding the role of interparticle necking on thermal conduction. The model is extended to three dimensions, and then employed to study the influence of particle size and porosity on transport characteristics of interest to heat pipe designers. It is observed that permeability is relatively independent of sintering conditions, but depends on porosity and particle size - as porosity or particle size increases, permeability increases and vice versa. Conversely, effective thermal conductivity is observed to increase steadily as a function of increasing sintering iterations (time) or pixels migrated per iteration (temperature). Similar observations were made for cases with constant porosity and a single particle size. Further, a realistic case of a distribution of particle sizes, indicative of an actual pre-sintered compact, was also investigated, and permeability for this case was observed to be very well predicted $(<15 \%$ maximum difference) via the Kozeny-Carman correlation.

\section{Acknowledgment}

The authors acknowledge support for this work from industry members of the Cooling Technologies Research Center, an NSF
Industry/University Cooperative Research Center at Purdue University.

\section{Nomenclature}

$A, d \mathbf{A}=$ area

$C(T)=$ coefficient in neck growth model

$C_{E}=$ Ergun's coefficient

$D, D_{p}=$ particle diameter

$\mathbf{J}=$ heat flux

$k_{\text {eff }}=$ effective thermal conductivity

$K=$ permeability

$m, n=$ exponents in neck growth model

$n=$ sintering parameter

$P=$ Pressure

$t=$ time

$T=$ temperature

$U=$ velocity

$V_{T}=$ volume of the sample

$x=$ sintering neck diameter

\section{Greek Symbols}

$$
\begin{aligned}
& \varepsilon=\text { porosity } \\
& \rho=\text { density } \\
& \mu=\text { viscosity }
\end{aligned}
$$

\section{Subscripts}

$$
\begin{aligned}
\text { eff } & =\text { effective } \\
p & =\text { particle } \\
\text { sf } & =\text { solid-fluid }
\end{aligned}
$$

\section{References}

[1] Krishnan, S., Garimella, S. V., Chrysler, G. M., and Mahajan, R. V., 2007, "Towards a Thermal Moore's Law," IEEE Trans. Adv. Packag., 30(3), pp. 462-474.

[2] Weibel, J. A., Garimella, S. V., and North, M. T., 2010, "Characterization of Evaporation and Boiling From Sintered Powder Wicks Fed by Capillary Action," Int. J. Heat Mass Transfer, 53(19-20), pp. 4204-4215.

[3] Wang, D., Yu, E., and Przekwas, A., 1999 "A Computational Study of Two Phase Jet Impingement Cooling of an Electronic Chip," Proceedings of Fifteenth Annual IEEE Semiconductor Thermal Measurement and Management Symposium, SEMI-THERM, Piscataway, NJ, pp. 10-15.

[4] Harichian, T., and Garimella, S. V., 2013, Microchannel Heat Sinks for Electronics Cooling, World Scientific, Singapore.

[5] Faghri, A., 1995, Heat Pipe Science and Technology, Taylor \& Francis Washington, DC

[6] Dunn, P. D., and Reay, D. A., 1973, "The Heat Pipe," Phys. Technol., 4 p. 187.

[7] Garimella, S. V., Joshi, Y. K., Bar-Cohen, A., Mahajan, R., Toh, K. C., Carey, V. P., Baelmans, M., Lohan, J., Sammakia, B., and Andros, F., 2003, "Thermal Challenges in Next Generation Electronic Systems-Summary of Panel 
Presentations and Discussions," IEEE Trans. Compon. Packag. Technol., 25(4), pp. 569-575.

[8] Dullien, F. A. L., 1979, Porous media, Fluid Transport and Pore Structure, Academic, New York.

[9] Bodla, K. K., Murthy, J. Y., and Garimella, S. V., 2012, "Direct Simulation of Thermal Transport Through Sintered Wick Microstructures," ASME J. Heat Transfer, 134(1), p. 012602.

[10] Bodla, K. K., Murthy, J. Y., and Garimella, S. V., 2013, "Evaporation Analysis in Sintered Wick Microstructures," Int. J. Heat Mass Transfer, 61, pp. 729-741.

[11] Bodla, K. K., Weibel, J. A., and Garimella, S. V., "Advances in Fluid and Thermal Transport Property Analysis and Design of Sintered Porous Wick Microstructures," ASME J. Heat Transfer, 135(6), p. 061202.

[12] German, R. M., 1996, Sintering Theory and Practice, John Wiley and Sons, New York.

[13] Wakai, F., 2006, "Modeling and Simulation of Elementary Processes in Ideal Sintering," J. Am. Ceram. Soc., 89(5), pp. 1471-1484.

[14] Wakai, F., and Aldinger, F., 2003, "Sintering Through Surface Motion by the Difference in Mean Curvature," Acta Mater., 51(14), pp. 4013-4024.

[15] Kumar, V., 2011, "Simulations and Modeling of Unequal Sized Particles Sintering," Ph.D. thesis, University of Utah, Salt Lake City, UT.

[16] Martin, C. L., Schneider, L. C. R., Olmos, L., and Bouvard, D., 2006, "Discrete Element Modeling of Metallic Powder Sintering," Scr. Mater., 55(5), pp. 425-428.

[17] Tikare, V., Braginsky, M., Bouvard, D., and Vagnon, A., 2010, "Numerical Simulation of Microstructural Evolution During Sintering at the Mesoscale in a 3D Powder Compact," Comput. Mater. Sci., 48(2), pp. 317-325.

[18] Braginsky, M., Tikare, V., and Olevsky, E., 2005, "Numerical Simulation of Solid State Sintering,” Int. J. Solids Struct., 42(2), pp. 621-636.
[19] Tikare, V., Braginsky, M., and Olevsky, E. A., 2003, "Numerical Simulation of Solid-State Sintering: I, Sintering of Three Particles," J. Am. Ceram. Soc. 86(1), pp. 49-53.

[20] Pimienta, P. J. P., Garboczi, E. J., and Carter, W. C., 1992, “Cellular Automaton Algorithm for Surface Mass Transport Due to Curvature Gradients. Simulations of Sintering," Comput. Mater. Sci., 1(1), pp. 63-77.

[21] Bentz, D. P., Pimienta, P. J. P., Garboczi, E. J., and Carter, W. C., 1991, "Cellular Automaton Simulations of Surface Mass Transport Due to Curvature Gradients: Simulations of Sintering in 3-Dimensions," Synth. Process. Ceram. Sci. Issue, 249, pp. 413-418.

[22] Bodla, K. K., Murthy, J. Y., and Garimella, S. V., 2010, "MicrotomographyBased Simulation of Transport through Open-Cell Metal Foams," Numer. Heat Transfer, Part A, 58(7), pp. 527-544.

[23] Brakke, K. A., 1992, “The Surface Evolver," Exp. Math., 1, pp. 141-165.

[24] Vicsek, T., 1984, "Pattern Formation in Diffusion-Limited Aggregation," Phys. Rev. Lett., 53(24), pp. 2281-2284.

[25] Bohn, R. B., and Garboczi, E. J., 2003, "User Manual for Finite Element Difference Programs: A Parallel Version of NISTIR 6269," National Institute of Standards and Technology Internal Report No. 6997, Gaithersburg, MD.

[26] Alexander, B. H., and Balluffi, R. W., 1957, "The Mechanism of Sintering of Copper," Acta Metall., 5(11), pp. 666-677.

[27] Bentz, D. P., 2000, "CEMHYD3D: A Three-Dimensional Cement Hydration and Microstructure Development Modelling Package. Version 2.0," National Institute of Standards and Technology Interagency Report No. 7232, Gaithersburg, MD.

[28] User's Guide for FLuENT 6.0, 2002, Fluent Inc., Lebanon, NH.

[29] Espinosa, F. A. D., Peters, T. B., and Brisson, J. G., 2012, "Effect of Fabrication Parameters on the Thermophysical Properties of Sintered Wicks for Heat Pipe Applications," Int. J. Heat Mass Transfer, 55, pp. 7471-7486. 